\title{
First Case Report of the Antemortem Diagnosis of Nonbacterial Thrombotic Endocarditis of a Mechanical Prosthetic Valve
}

\author{
Hideki Yasutake ${ }^{1}$, Yasuo Sugano ${ }^{1}$, Yoshihiko Ikeda ${ }^{2}$, Takahiro Ohara ${ }^{1}$, Takuya Hasegawa ${ }^{1}$, \\ Hideaki Kanzaki ${ }^{1}$ and Toshihisa Anzai ${ }^{1}$
}

\begin{abstract}
A 67-year-old man with a history of aortic mechanical valve replacement exhibited an abnormal mass on the sewing ring of the prosthesis on echocardiography. Despite receiving strong anticoagulation, he developed acute cerebral infarction due to the formation of emboli resulting from the thrombus and underwent urgent re-aortic valve replacement. Based on the microscopic findings of the resected mass, he was finally diagnosed as having nonbacterial thrombotic endocarditis (NBTE) of the mechanical prosthetic valve, which was thought to be associated with colorectal cancer. We herein report the first known case of an antemortem diagnosis of NBTE on a mechanical heart valve.
\end{abstract}

Key words: nonbacterial thrombotic endocarditis, prosthetic valve, malignancy, diagnosis

(Intern Med 55: 255-257, 2016)

(DOI: 10.2169/internalmedicine.55.5470)

\section{Introduction}

Nonbacterial thrombotic endocarditis (NBTE) occurs most commonly on the cardiac valves of patients with malignant or collagen disease. NBTE is typically diagnosed at autopsy; however, in some cases, an antemortem diagnosis is made as a result of the manifestations of recurrent systemic embolization. NBTE is considered to develop on native valves with endothelial injury due to a hypercoagulable state. However, the formation of NBTE on a prosthetic valve, in which the developmental process might differ from that observed on native valves, has not been previously described. We herein report a case of NBTE growing on the prosthetic aortic valve of a patient with colorectal cancer.

\section{Case Report}

A 67-year-old man was admitted to our hospital for a detailed examination and treatment of an abnormal mass on a prosthetic aortic valve. Ten years previously, he had under- gone the Bentall operation with a 25-mm ATS mechanical valve for aortic valve insufficiency due to annuloaortic ectasia and mitral annuloplasty for concomitant mitral valve insufficiency. He subsequently underwent implantation of a permanent DDD pacemaker for postoperative refractory atrioventricular block and was thereafter followed up annually with transthoracic echocardiography (TTE). One year before admission, a $0.6 \mathrm{~cm}$ by $0.3 \mathrm{~cm}$ echo-dense mass was found on the sewing ring of his prosthetic aortic valve using TTE. The mass on the mechanical prosthesis appeared to be quite small and it was not regarded as being abnormal. Follow-up TTE performed the next year revealed an increase in size of the abnormal mass to $1.2 \mathrm{~cm}$ by $0.9 \mathrm{~cm}$, and the lesion was mobile, without causing interference with prosthetic valve motion (Fig. 1). He was therefore admitted to our hospital for a further investigation.

The patient had no symptoms and showed normal physical examination findings. A chest $\mathrm{X}$-ray demonstrated no pulmonary congestion or pleural fluid. Laboratory tests showed no elevation of the white blood cell count or Creactive protein level, although the D-dimer level was

${ }^{1}$ Department of Cardiovascular Medicine, National Cerebral and Cardiovascular Center, Japan and ${ }^{2}$ Department of Pathology, National Cerebral and Cardiovascular Center, Japan

Received for publication March 28, 2015; Accepted for publication May 12, 2015

Correspondence to Dr. Yasuo Sugano, ysugano@ncvc.go.jp 


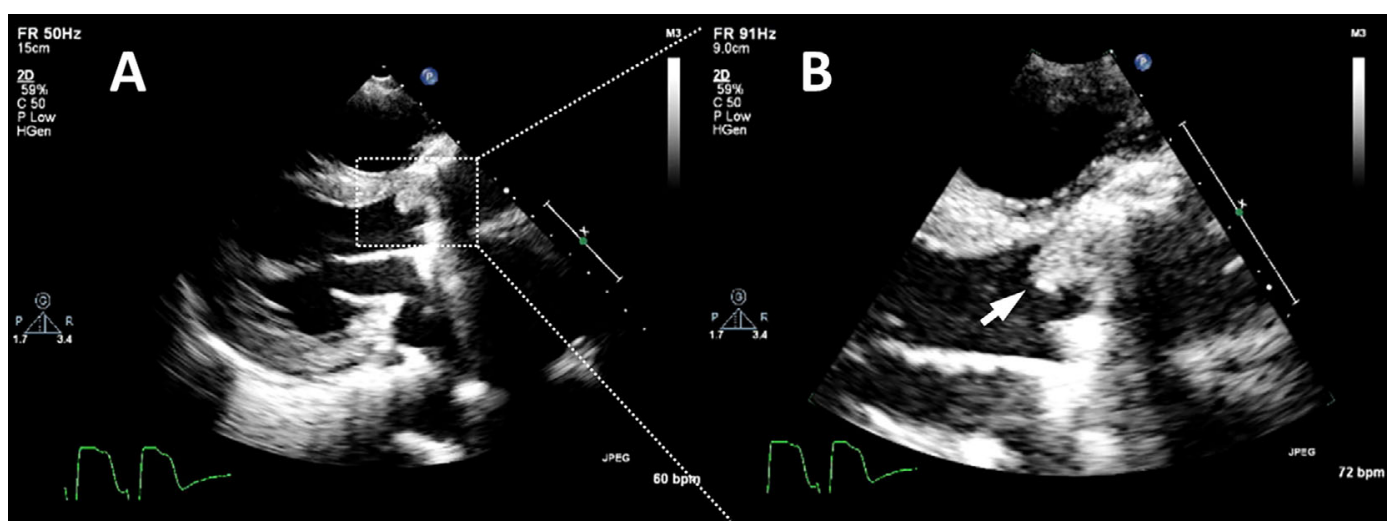

Figure 1. Still transthoracic echocardiographic image. A: Abnormal mass on the prosthetic aortic valve in the parasternal long-axis view. B: Magnified view showing a mobile vegetation-like echo (arrow) at the sewing ring of the prosthetic aortic valve.
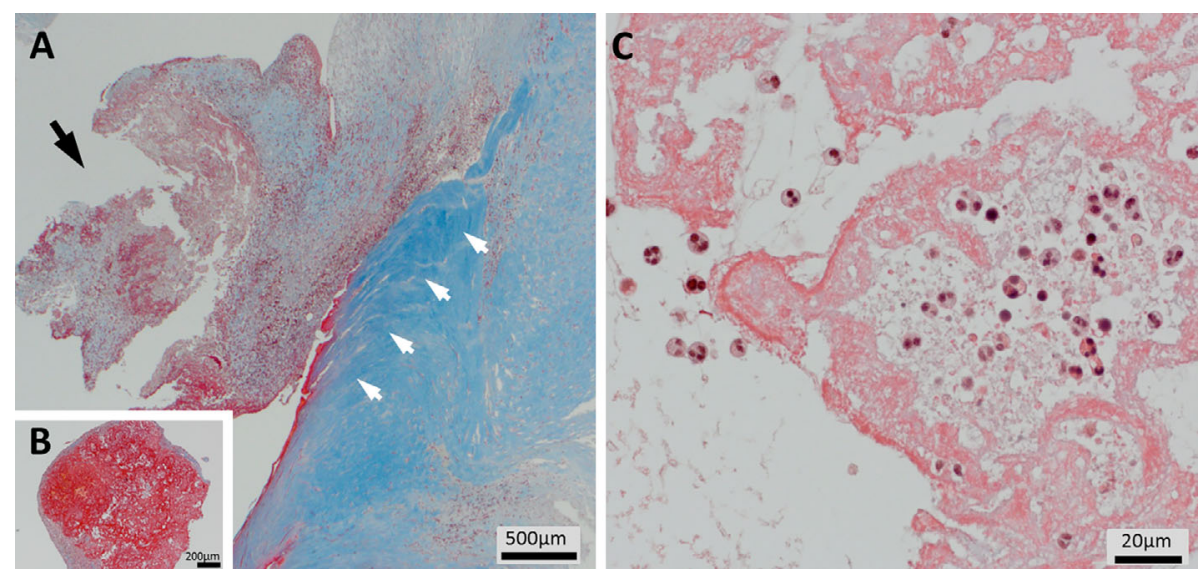

Figure 2. Histopathological findings of vegetation. A, B: Low-power view of a Masson trichromestained section of the root of the area of vegetation (A) and a part of the thrombus (B). The thrombus was attached to the part indicated by the black arrow. The site of vegetation was connected to the pannus (white arrows). High-power view of a Hematoxylin and Eosin staining section showing the area of vegetation to be composed of fibrin and neutrophils without bacteria (C).

slightly elevated at $1.3 \mu \mathrm{g} / \mathrm{mL}$. Serial blood cultures were negative, and infective endocarditis was thus considered to be unlikely. A brain CT scan showed multiple old cerebral infarcts, possibly due to microemboli. Although the patient had been treated with warfarin under proper control with a prothrombin time (PT)-international normalized ratio (INR) between 2.0 and 3.0, he was suspected of having a thrombus on the mechanic aortic valve. He was then treated with strong anticoagulation by adding intravenous heparin to oral warfarin. The PT-INR value was 2.02 under therapy with 5 $\mathrm{mg}$ of warfarin on admission. The dose of warfarin was then increased up to $7 \mathrm{mg}$, which resulted in a PT-INR of 2.66 on the 12th hospital day.

On the 19th day of hospitalization, the patient experienced the sudden onset of right hemiparesis. An emergency brain CT scan revealed no evidence of acute cerebral infarction; however, judging from the clinical findings, he was diagnosed as having acute cerebral infarction, presumably caused by emboli originating from the thrombus on the prosthetic valve. He subsequently underwent urgent re-aortic valve replacement with a $23-\mathrm{mm}$ Trifecta biological valve. Pannus formation was seen, with tissue connected macroscopically with the sewing ring of the resected prosthetic valve and a $2.0 \times 1.0 \mathrm{~cm}$ mass attached on the pannus. Pathologically, the mass was composed of platelet aggregates and fibrin and devoid of signs of intense inflammation with bacteria, except for scattered neutrophils, features compatible with NBTE (Fig. 2). A culture test of the resected tissue was negative for bacteria. The patient was suspected of having malignant gastrointestinal disease based on an increased carcinoembryonic antigen (CEA) level of $11.1 \mathrm{ng} / \mathrm{mL}$ and positive fecal occult blood test. Diseases associated with NBTE, such as rheumatoid arthritis, systemic lupus erythematosus and Behçet's disease, were ruled out, with all collagen disease-associated serological values, such as antinuclear antibody (ANA), rheumatoid factor (RF) and antiphospholipid antibody (aPL) titers, less than the sensitivity limits. After the valve operation, the patient underwent colonoscopy, 
which showed advanced descending colorectal cancer without distant metastasis, after which he received successful left hemi-colectomy. A pathological examination revealed that the colorectal cancer was tubular adenocarcinoma and invaded beyond the middle layer of the colon.

\section{Discussion}

NBTE is defined as a disease presenting with vegetation, devoid of intense inflammation with bacteria, and is generally associated with underlying diseases such as malignancy and autoimmune disorders and characterized by fatal recurrent embolic strokes $(1,2)$. There have only been a few antemortem reports of NBTE, as most areas of vegetation are small and not easily detected on echocardiography (3). Thus far, no cases of NBTE affecting a prosthetic valve have been reported in the literature. In the current case, we made an antemortem diagnosis of NBTE on a prosthetic valve and demonstrated pathologically that the lesion was attached to the pannus.

The pathogenesis of NBTE is not fully elucidated, although it is believed to involve excessive immune activity caused by malignancy or autoimmune disease, which plays a role in the progression of vegetation (4). Increased cytokines, such as tumor necrosis factor and interleukin-1, derived from immunologically active cells cause endothelial damage, initiating platelet aggregation on the valve, followed by the development of a hypercoagulable state based on the background disease, which facilitates nonbacterial formation at the site $(1,5)$. Therefore, mechanical valves, which have no endothelial tissue on their surface, are considered to be inappropriate sites for the onset of NBTE. However, the development of pannus tissue on a prosthetic valve can be the basis for the occurrence of NBTE. Pannus formation, consisting of the deposition of collagen and elastic tissue, endothelial cells, chronic inflammatory cells and myofibroblasts, is known to function as an inflammatory bioreactor against prosthetic valves (6).

Typical pathological features of NBTE include the composition of blood and aggregated platelet thrombi devoid of inflammatory reactions with bacteria $(7,8)$. Although there was scattered accumulation of neutrophils in this case, the features of the resected site of vegetation were compatible with NBTE, with abundant platelet aggregation and fibrin deposition. The scarcity of inflammation may be associated with the recurrent thromboembolic profile of NBTE, with a tendency to fragment and not grow in size (9).

Treatment with intravenous heparin is known to be effec- tive for such patients, and surgical resection or valve replacement is not selected in most cases because of the high incidence of recurrence of NBTE, unless the underlying cause is corrected (10). In the present case, we considered urgent re-aortic valve replacement to be necessary before the diagnosis of rectal cancer based on the embolic stroke episode that occurred during intravenous heparin administration, with a visible mobile area of vegetation on the prosthetic aortic valve. Immediately after the valve operation, the patient successfully underwent colectomy for the colorectal cancer.

\section{The authors state that they have no Conflict of Interest (COI).}

\section{References}

1. el-Shami K, Griffiths E, Streiff M. Nonbacterial thrombotic endocarditis in cancer patients: pathogenesis, diagnosis, and treatment. The Oncologist 12: 518-523, 2007.

2. Asopa S, Patel A, Khan OA, Sharma R, Ohri SK. Non-bacterial thrombotic endocarditis. Eur J Cardiothorac Surg 32: 696-701, 2007.

3. Edoute Y, Haim N, Rinkevich D, Brenner B, Reisner SA. Cardiac valvular vegetations in cancer patients: a prospective echocardiographic study of 200 patients. Am J Med 102: 252-258, 1997.

4. Katsouli A, Massad MG. Current issues in the diagnosis and management of blood culture-negative infective and non-infective endocarditis. Ann Thorac Surg 95: 1467-1474, 2013.

5. Carey MJ, Rodgers GM. Disseminated intravascular coagulation: clinical and laboratory aspects. Am J Hematol 59: 65-73, 1998.

6. Teshima H, Hayashida N, Yano H, et al. Obstruction of St Jude Medical valves in the aortic position: histology and immunohistochemistry of pannus. J Thorac Cardiovasc Surg 126: 401-407, 2003.

7. Allen AC, Sirota JH. The morphogenesis and significance of degenerative verrucal endocarditis (terminal endocarditis, endocarditis simplex, nonbacterial thrombotic endocarditis). Am J Pathol 20: 1025-1055, 1944.

8. Eiken PW, Edwards WD, Tazelaar HD, McBane RD, Zehr KJ. Surgical pathology of nonbacterial thrombotic endocarditis in 30 patients, 1985-2000. Mayo Clin Proc 76: 1204-1212, 2001.

9. Singhal AB, Topcuoglu MA, Buonanno FS. Acute ischemic stroke patterns in infective and nonbacterial thrombotic endocarditis: a diffusion-weighted magnetic resonance imaging study. Stroke 33: 1267-1273, 2002.

10. Lee AY, Levine MN, Baker RI, et al. Randomized comparison of low molecular-weight heparin versus oral anticoagulant therapy for the prevention of recurrent venous thromboembolism in patients with cancer (CLOT) investigators. Low-molecular-weight heparin versus a coumarin for the prevention of recurrent venous thromboembolism in patients with cancer. N Engl J Med 349: 146-153, 2003.

(C) 2016 The Japanese Society of Internal Medicine http://www.naika.or.jp/imonline/index.html 